\title{
Implementation and Application of the Relativistic Equation of Motion Coupled-cluster Method for the Excited States of Closed-shell Atomic Systems
}

\author{
D. K. Nandy *, Yashpal Singh and B. K. Sahoo ${ }^{\dagger}$ \\ Theoretical Physics Division, Physical Research Laboratory, Navrangpura, Ahmedabad 380009, India
}

(Dated: Received date; Accepted date)

\begin{abstract}
We report the implementation of equation-of-motion coupled-cluster (EOMCC) method in the four-component relativistic framework with the spherical atomic potential to generate the excited states from a closed-shell atomic configuration. This theoretical development will be very useful to carry out high precision calculations of varieties of atomic properties in many atomic systems. We employ this method to calculate excitation energies of many low-lying states in a few Nelike highly charged ions, such as Cr XV, Fe XVII, Co XVIII and Ni XIX ions, and compare them against their corresponding experimental values to demonstrate the accomplishment of the EOMCC implementation. The considered ions are apt to substantiate accurate inclusion of the relativistic effects in the evaluation of the atomic properties and are also interesting for the astrophysical studies. Investigation of the temporal variation of the fine structure constant $(\alpha)$ from the astrophysical observations is one of the modern research problems for which we also estimate the $\alpha$ sensitivity coefficients in the above ions.
\end{abstract}

PACS numbers: 31.10.+z, 31.15.A-, 31.15.ag, 31.15.ap

\section{INTRODUCTION}

With the advent of sophisticated advanced technologies, modern research in atomic physics demands many high precision atomic calculations. Some of the prominent examples in this context are, studies of parity nonconservation (PNC) and permanent electric dipole moments (EDMs) [1, 2], estimation of the uncertainties for the frequency standard measurements [3-5], probing variation of the fine structure constant [6, 7], extracting nuclear charge radii and nuclear moments [810], providing atomic data for the astrophysical investigations [11, 12] et cetera. In the last two decades, the coupled-cluster (CC) method for the single valence systems in the four-component relativistic framework have been extensively employed for the above mentioned research problems with a great success [13 16]. In contrast, such CC methods are scanty for calculating the excited state properties of the systems with closed-shell configurations as far as the four-component relativistic approach with the explicit form of the spherical atomic potentials are concerned. There have been development of CC methods in the Fock-space formalism to calculate these states 17-19], however such approaches suffer from two serious problems: (i) It increases the computational complexity when applied to estimate the matrix elements of an operator and (ii) It yields intruder state problem while increasing the size of the model space [20]. On the other hand, the low-lying odd parity forbidden transitions among the singlet states of the systems like $\mathrm{Mg}$, $\mathrm{Ca}, \mathrm{Sr}, \mathrm{Al}^{+}, \mathrm{In}^{+}, \mathrm{Hg}^{+}, \mathrm{Yb}$ etc. are considered for the atomic clock experiments [27 31]. Similarly, atomic sys-

\footnotetext{
*Email: dillip@prl.res.in

${ }^{\dagger}$ Email: bijaya@prl.res.in
}

tems like $\mathrm{Xe}, \mathrm{Ba}, \mathrm{Ra}, \mathrm{Yb}, \mathrm{Hg}, \mathrm{Rn}$, etc. have been considered for the PNC and EDM studies [21 26] for which high precision calculations in the relativistic method are indispensable. Coincidently, the excited states involved in the above research problems can be created by exciting one electron from an occupied orbital (hole (h)) to a unoccupied orbital (particle (p)) which is customarily referred to as $1 \mathrm{~h}-1 \mathrm{p}$ excitation in the literature.

Among various popular many-body methods, equation-of-motion coupled-cluster (EOMCC) theory is one of the better suited methods to obtain the excited states [20, 32]. This method is formulated in the similar way as the excitation operators defined for the configuration-interaction (CI) method, however the excitations are carried out with respect to the exact state in contrast to the model reference state of the CI method. Uniqueness of this approach is that the energy differences between the atomic states are estimated directly by casting the Schrödinger equations in a particular form. Many non-relativistic calculations on the ionization potentials (IPs), electron affinities (EAs) and excitation energies (EEs) in different atomic and molecular systems have been reported in the EOMCC framework 20, 32 35. Recently, this method has been developed to determine the first and second IPs of many closed-shell systems using the atomic integrals in the four-component relativistic mechanics 36, 37]. However, all the above EOMCC calculations are carried out using the molecular codes considering special group symmetry properties. In this work, we discuss about the implementation of the EE determining EOMCC method (referred to as EE-EOMCC method) based on the fourcomponent relativistic mechanics and expressing the atomic potentials explicitly in the spherical polar coordinates. Also, all the physical operators are represented in terms of the Racah angular momentum operators for which we make use of the reduced matrix elements in 
order to reduce the computational scalability.

In order to corroborate the successful implementation of the excited states determining EE-EOMCC method, we calculate EEs of transitions among the low-lying states in few Ne-like systems that are of immense astrophysical interest. Transition lines in the range 10.5-21.2 $A$ of Fe XVII are observed in the solar corona 38 -41]. It is also found from the stellar binary Capella observation that Fe XVII is one of the important constituents in the stellar corona [42] and transition lines from this ion have also been observed from various astrophysical objects by many others [43 48]. The above lines and transitions from Co XVIII and Ni XIX are also very important for the astrophysical and tokamak plasma studies [40, 49]. The bottom line for the referral of these astrophysical observation is that the above transition lines could be of potential candidates for probing temporal variation of the fine structure constant $(\alpha)$. In our recent works, we have provided data to probe $\alpha$ variation by employing a single reference CC method formulated in the Fockspace approach for the $\mathrm{F}$ - and $\mathrm{Cl}-$ like $\mathrm{Cr}, \mathrm{Fe}$ and $\mathrm{Ni}$ ions [50, 51]. We intend, here, to estimate the sensitivity coefficients in the considered ions for the investigation of $\alpha$ variation whose magnitudes would magnitudes would gauge the significance of the relativistic effects and can be undertaken for their detection.

\section{PERCEPTION OF $\alpha$ VARIATION SENSITIVITY COEFFICIENTS}

Probing different absorption systems, associated with the quasi-stellar objects like quasars, can provide useful information regarding the speculated temporal variability of $\alpha$. These absorption systems that are present at different redshifts contain many metallic and non-metallic ions of various elements. A small fraction of the absorption systems, that are detected through the analysis of the quasars spectra, could be intrinsically associated to the quasars themselves. Investigations of the broad absorptions line systems (BALs) of this region reveal that the chemical compositions are usually from the highly ionized species [52, 53]. Next to BALs, the most important regions are the intervening absorption systems which can be further classified into various regions depending upon the column density of the neutral hydrogen $(\mathrm{H})$ atoms $[54$ 56]. The absorptions lines that are coming out of these systems are red-shifted due to the cosmological expansion of the universe and are related to the cosmological redshift parameter $(z)$ by the following relation

$$
\lambda_{z}=\lambda_{\text {rest }}(1+z),
$$

where $\lambda_{\text {rest }}$ is the wavelength at the time of emission in the rest frame of the absorption system. With the precise knowledge of the redshift of an absorption line, one can extract the information about the subtle temporal variation in $\alpha$ after the cautious consideration of the systematic uncertainties associated with the observation [57]. These absorption lines are observed with the advanced telescope such as high-resolution Echelle Spectrograph (HIRES) at the Keck Observatory or the UVVisual Echelle Spectrograph (UVES) at the ESO Very Large Telescope (VLT) for the investigation of variation in $\alpha[58,59]$.

The anticipated tiny variation in $\alpha$ from the present laboratory value $\alpha_{0}$ can be inferred by combining the calculated relativistic sensitivity coefficients ( $q \mathrm{~s})$ of different atomic transitions with the observed spectral lines from the quasars 7]. Since the energy of an atomic level scales at the order of $\alpha^{2}$ in the relativistic theory, the frequency of an atomic transition will depend on the value of $\alpha$ at a given time. The relativistic corrections to the energy levels of a multi-electron atom can be expressed as [60]

$$
\Delta=-\frac{Z_{a}^{2}}{2} \frac{(Z \alpha)^{2}}{\nu^{3}}\left(\frac{1}{J+1 / 2}-\frac{Z_{a}}{Z \nu}\left[1-\frac{Z_{a}}{4 Z}\right]\right),
$$

with $Z$ is the atomic number, $J$ is the angular momentum of the state and $\nu$ and $Z_{a}$ are the effective principal quantum number and effective atomic number, respectively, of an outer electron due to the screening effects of the inner core electrons. It has been shown that instead of considering two transitions from a particular atomic system (alkali doublet (AD) method), it is advantageous to compare as many as transitions from a number of systems (many-multiplet (MM) method) to yield an order of magnitude improvement in the detection of change in $\alpha$ value $(\Delta \alpha)$ from the observations [61 63]. Generally, one compares the measured velocity profile in the MM method to infer tiny shifts in the transitions, having different magnitudes of $q$ parameters, to obtain stringent value of $\frac{\Delta \alpha}{\alpha}$ from a best possible fit. In this method the change in the transition frequency between two states of an atomic system with respect to an arbitrary variation in $\alpha$, quantified as $x=\left(\frac{\alpha}{\alpha_{0}}\right)^{2}-1$, can be given by

$$
\omega\left(\alpha^{2}\right) \approx \omega\left(\alpha_{0}^{2}\right)+q x,
$$

such that $q=\left.\frac{d \omega}{d\left(\alpha^{2}\right)}\right|_{x=0}$ corresponds to rate of change of $\omega$, which is independent of $x$, and known as the sensitivity coefficient for $\alpha$ variation. In the MM method, the commonly used relation for the extraction of change in $\alpha$ is given by

$$
\frac{\Delta v}{c}=-\frac{2 q}{\omega\left(\alpha_{0}^{2}\right)} \frac{\Delta \alpha}{\alpha},
$$

where $c$ is the velocity of light, $\Delta v$ corresponds to the change in the velocity profile of the absorption lines that are related to the wavelengths of the atomic transitions. Therefore for probing $\alpha$ variation using the MM method, it is imperative to find out $q$ parameters in many possible transitions of the atomic systems that are highly abundant in the astrophysical objects like the considered ions in the present work. 


\section{RELATIVISTIC ATOMIC INTEGRALS}

For the present calculation, we consider following relativistic Dirac-Coulomb (DC) Hamiltonian which is rescaled with respect to the rest mass energy of the electrons

$$
H=\sum_{i}\left[c \boldsymbol{\alpha}_{i} \cdot \mathbf{p}_{i}+\left(\beta_{i}-1\right) c^{2}+V_{n u c}\left(r_{i}\right)+\sum_{j>i} \frac{1}{r_{i j}}\right]
$$

where $\boldsymbol{\alpha}_{i}$ and $\beta_{i}$ are the usual Dirac matrices, $V_{n u c}\left(r_{i}\right)$ is the nuclear potential and $\frac{1}{r_{i j}}=\frac{1}{\left|\mathbf{r}_{i}-\mathbf{r}_{j}\right|}$ is the interelectronic Coulombic repulsion potential. The nuclear potential is evaluated by considering the Fermi-charge distribution of the nuclear density as given by

$$
\rho_{n u c}(r)=\frac{\rho_{0}}{1+e^{(r-b) / d}}
$$

where the parameter ' $b$ ' is the half-charge radius as $\rho_{n u c}(r)=\rho_{0} / 2$ for $r=b$ and ' $d$ ' is related to the skin thickness which are evaluated by

$$
\begin{aligned}
d & =2.3 / 4(\ln 3) \\
\text { and } \quad b & =\sqrt{\frac{5}{3} r_{r m s}^{2}-\frac{7}{3} d^{2} \pi^{2}}
\end{aligned}
$$

with $r_{r m s}$ is the root mean square radius of the nucleus.

In the relativistic quantum mechanics, the fourcomponent Dirac wave function for a single electron is expressed by

$$
|\phi(r)\rangle=\frac{1}{r}\left(\begin{array}{cc}
P(r) & \chi_{\kappa, m}(\theta, \phi) \\
i Q(r) & \chi_{-\kappa, m}(\theta, \phi)
\end{array}\right)
$$

where $P(r)$ and $Q(r)$ are the large and small components of the wave function respectively. The angular components have the following form

$$
\chi_{\kappa, m}(\theta, \phi)=\sum_{\sigma= \pm \frac{1}{2}} C(l \sigma j ; m-\sigma, \sigma) Y_{l}^{m-\sigma}(\theta, \phi) \phi_{\sigma}
$$

where $C(l \sigma j ; m-\sigma, \sigma)$ is Clebsch-Gordan (Racah) coefficient, $Y_{l}^{m-\sigma}(\theta, \phi)$ represents normalized spherical harmonics, $\phi_{\sigma}$ serves as the Pauli two-component spinors and the relativistic quantum number $\kappa=-\left(j+\frac{1}{2}\right) a$ embodies the total and orbital quantum numbers $j$ and $l=j-\frac{a}{2}$.

With the defined Dirac-Fock (DF) potential as

$$
U\left|\phi_{j}\right\rangle=\sum_{a=1}^{o c c}\left\langle\phi_{a}\left|\frac{1}{r_{j a}}\right| \phi_{a}\right\rangle\left|\phi_{j}\right\rangle-\left\langle\phi_{a}\left|\frac{1}{r_{a j}}\right| \phi_{j}\right\rangle\left|\phi_{a}\right\rangle,
$$

summed over all the occupied orbitals occ, the DF wave function $\left(\left|\Phi_{0}\right\rangle\right)$ for a close-shell atomic system is obtained by solving the equation

$$
H_{D F}\left|\Phi_{0}\right\rangle=E_{D F}^{(0)}\left|\Phi_{0}\right\rangle
$$

which in terms of the single particle orbitals are given by

$$
\sum_{i}\left[h_{0}\left|\phi\left(r_{i}\right)\right\rangle=\epsilon_{i}\left|\phi\left(r_{i}\right)\right\rangle\right]
$$

for the DF Hamiltonian

$$
\begin{aligned}
H_{D F} & =\sum_{i}\left[c \boldsymbol{\alpha}_{i} \cdot \mathbf{p}_{i}+\left(\beta_{i}-1\right) c^{2}+V_{n u c}\left(r_{i}\right)+U\left(r_{i}\right)\right] \\
& =\sum_{i} h_{0}\left(r_{i}\right)
\end{aligned}
$$

where $h_{0}$ is the single particle Fock operator.

We express $\left|\phi_{n, \kappa}(r)\right\rangle$, with the principal quantum number $n$ and angular quantum number $\kappa$, of an electron orbital as linear combination of Gaussian type of orbitals (GTOs) to obtain the DF orbitals. In the spherical polar coordinates, it is given by

$\left|\phi_{n, \kappa}(r)\right\rangle=\frac{1}{r} \sum_{\nu}\left(\begin{array}{cc}C_{n, \kappa}^{L} N_{L} f_{\nu}(r) & \chi_{\kappa, m} \\ i C_{n,-\kappa}^{S} N_{S}\left(\frac{d}{d r}+\frac{\kappa}{r}\right) f_{\nu}(r) & \chi_{-\kappa, m}\end{array}\right)$

where $C_{n, \kappa} \mathrm{s}$ are the expansion coefficients, $N_{L(S)}$ are the normalization constants for the large (small) components of the wave function and $f_{\nu}(r)=r^{l} e^{-\eta_{\nu} r^{2}}$ are the GTOs with the suitably chosen parameters $\eta_{\nu}$ for orbitals of different angular momentum symmetries. For the exponents, we use the even tempering condition $\eta_{\nu}=\eta_{0} \zeta^{\nu-1}$ with two parameters $\eta_{0}$ and $\zeta$. It can be noticed in the above expression that the large and small components of the wave function satisfy the kinetic balance condition. The orbitals are finally obtained by executing a self-consistent procedure to solve the following eigenvalue form of the DF equation

$$
\sum_{\nu}\left\langle f_{i, \mu}\left|h_{0}\right| f_{i, \nu}\right\rangle c_{i \nu}=\epsilon_{i} \sum_{\nu}\left\langle f_{i, \mu} \mid f_{i, \nu}\right\rangle c_{i \nu}
$$

which is in the matrix form given by

$$
\sum_{\nu} F_{\mu \nu} c_{i \nu}=\epsilon_{i} \sum_{\mu \nu}\left\langle f_{i, \mu} \mid f_{i, \nu}\right\rangle c_{i \nu}
$$

The above equation implies that the parity and the total angular momentum of an orbital are fixed which are the essential conditions to describe the mechanics in the spherical coordinates.

To retain the atomic spherical symmetry property in our calculations, the matrix form of the Coulomb interaction operator using the above single particle wave functions are expressed as

$$
\begin{aligned}
\left\langle\phi_{a} \phi_{b}\left|\frac{1}{r_{12}}\right| \phi_{c} \phi_{d}\right\rangle & =\int d r_{1}\left[P_{a}\left(r_{1}\right) P_{c}\left(r_{1}\right)+Q_{a}\left(r_{1}\right) Q_{c}\left(r_{1}\right)\right] \\
& \times \int d r_{2}\left[P_{b}\left(r_{2}\right) P_{d}\left(r_{2}\right)+Q_{b}\left(r_{2}\right) Q_{d}\left(r_{2}\right)\right] \\
& \times \sum_{k} \frac{r_{<}^{k}}{r_{>}^{k+1}} \times A n g,
\end{aligned}
$$


in which the $k$ multi-poles are determined by considering the triangle conditions $\left|j_{a}-j_{c}\right| \leq k \leq j_{a}+j_{c}$ and $\left|j_{b}-j_{d}\right| \leq k \leq j_{b}+j_{d}$ along with the additional restrictions over $k$ by multiplying a factor $\Pi\left(\kappa, \kappa^{\prime}, k\right)=$ $\frac{1}{2}\left[1-a a^{\prime}(-1)^{j+j^{\prime}+k}\right]$ that is finite only for $l+l^{\prime}+k=$ even. The angular momentum factor of the above expression is given by

$$
\begin{aligned}
A n g= & \delta\left(m_{a}-m_{c}, m_{d}-m_{d}\right) \Pi\left(\kappa_{a}, \kappa_{c}, k\right) \Pi\left(\kappa_{b}, \kappa_{d}, k\right) \\
& \times(-1)^{q} \sqrt{\left(2 j_{a}+1\right)\left(2 j_{b}+1\right)\left(2 j_{c}+1\right)\left(2 j_{d}+1\right)} \\
& \times\left(\begin{array}{ccc}
j_{a} & k & j_{c} \\
-m_{a} & q & m_{c}
\end{array}\right)\left(\begin{array}{ccc}
j_{b} & k & j_{d} \\
-m_{b} & -q & m_{d}
\end{array}\right) \\
& \times\left(\begin{array}{ccc}
j_{a} & k & j_{c} \\
\frac{1}{2} & 0 & -\frac{1}{2}
\end{array}\right)\left(\begin{array}{ccc}
j_{b} & k & j_{d} \\
\frac{1}{2} & 0 & -\frac{1}{2}
\end{array}\right),
\end{aligned}
$$

where $m_{j}$ is the azimuthal component of $j$. In order to minimize the computational efforts, we use the reduced matrix elements. Thus, we express

$$
\begin{aligned}
\left\langle\phi_{a} \phi_{b}\left|\frac{1}{r_{12}}\right| \phi_{c} \phi_{d}\right\rangle & =\delta\left(m_{a}-m_{c}, m_{d}-m_{b}\right) \sum_{k, q} \Pi\left(\kappa_{a}, \kappa_{c}, k\right) \\
& \times \Pi\left(\kappa_{b}, \kappa_{d}, k\right)(-1)^{j_{a}-m_{a}+j_{b}-m_{b}+k-q} \\
& \times\left(\begin{array}{ccc}
j_{a} & k & j_{c} \\
-m_{a} & q & m_{c}
\end{array}\right)\left(\begin{array}{ccc}
j_{b} & k & j_{d} \\
-m_{b} & -q & m_{d}
\end{array}\right) \\
& \times\left\langle a b\left\|\frac{1}{r_{i j}}\right\| c d\right\rangle,
\end{aligned}
$$

with the reduced matrix element

$$
\begin{aligned}
\left\langle a b\left\|\frac{1}{r_{i j}}\right\| c d\right\rangle & =(-1)^{j_{a}+j_{b}+k+1} \\
& \times \int d r_{1}\left[P_{a}\left(r_{1}\right) P_{c}\left(r_{1}\right)+Q_{a}\left(r_{1}\right) Q_{c}\left(r_{1}\right)\right] \\
& \times \int d r_{2}\left[P_{b}\left(r_{2}\right) P_{d}\left(r_{2}\right)+Q_{b}\left(r_{2}\right) Q_{d}\left(r_{2}\right)\right] \\
& \times \sqrt{\left(2 j_{a}+1\right)\left(2 j_{b}+1\right)\left(2 j_{c}+1\right)\left(2 j_{d}+1\right)} \\
& \times \frac{r_{<}^{k}}{r_{>}^{k+1}}\left(\begin{array}{ccc}
j_{a} & k & j_{c} \\
\frac{1}{2} & 0 & -\frac{1}{2}
\end{array}\right)\left(\begin{array}{ccc}
j_{b} & k & j_{d} \\
\frac{1}{2} & 0 & -\frac{1}{2}
\end{array}\right) \cdot(3.17)
\end{aligned}
$$

\section{RELATIVISTIC EE-EOMCC METHOD FOR ATOMS}

The starting point of our EOMCC method is the ground state wave function $\left(\left|\Psi_{0}\right\rangle\right)$ of a closed-shell system which in the $\mathrm{CC}$ formalism is expressed as

$$
\left|\Psi_{0}\right\rangle=e^{T}\left|\Phi_{0}\right\rangle
$$

where $\left|\Psi_{0}\right\rangle$ is the exact ground state and $\left|\Phi_{0}\right\rangle$ is the DF reference state taken in the anti-symmetrized form. We have restricted to only the singly and doubly excited configurations from $\left|\Phi_{0}\right\rangle$ in our calculations (CCSD method) by defining $T=T_{1}+T_{2}$, which in the second quantization notation are given by

$$
T_{1}=\sum_{a, p} a_{p}^{\dagger} a_{a} t_{a}^{p}, \quad \text { and } \quad T_{2}=\frac{1}{4} \sum_{a b, p q} a_{p}^{\dagger} a_{q}^{\dagger} a_{b} a_{a} t_{a b}^{p q}
$$
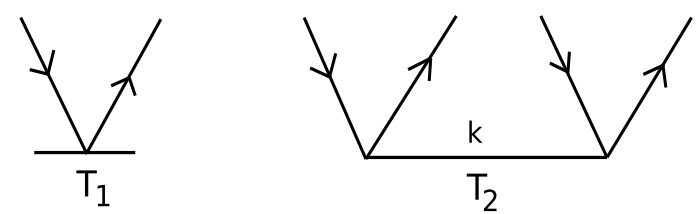

FIG. 1: Diamagnetic representations of the $T_{1}$ and $T_{2}$ excitation operators for the closed-shell CC method. $k$ is the rank of the $T_{2}$ operator.

where the subscripts $a, b$ and $p, q$ represent for the core and virtual orbitals, $a$ and $a^{\dagger}$ are the annihilation and creation operators, and $t_{a}^{p}$ and $t_{a b}^{p q}$ are the singly and doubly excited amplitudes. In a spherical coordinate system they are expressed as

$$
\left\langle j m_{j}\left|T_{1}\right| j^{\prime} m_{j}^{\prime}\right\rangle=(-1)^{j-m_{j}} \sum_{k, q}\left(\begin{array}{ccc}
j & k & j^{\prime} \\
-m_{j} & q & m_{j}^{\prime}
\end{array}\right)\left\langle j\left\|t_{1}^{k}\right\| j^{\prime}\right\rangle
$$

and

$$
\begin{aligned}
& \left\langle j_{a} m_{a} ; j_{b} m_{b}\left|T_{2}\right| j_{c} m_{c} ; j_{d} m_{d}\right\rangle=(-1)^{j_{a}-m_{a}+j_{b}-m_{b}} \\
& \times \sum_{k, q}(-1)^{k-q} \times\left(\begin{array}{ccc}
j_{a} & k & j_{c} \\
-m_{a} & q & m_{c}
\end{array}\right)\left(\begin{array}{ccc}
j_{b} & k & j_{d} \\
-m_{b} & -q & m_{d}
\end{array}\right) \\
& \times\left\langle j_{a} j_{b}|| t_{2}^{k}|| j_{c} j_{d}\right\rangle,
\end{aligned}
$$

where $\left\langle j\left\|t_{1}^{k}\right\| j^{\prime}\right\rangle$ and $\left\langle j_{a} j_{b}\left\|t_{2}^{k}\right\| j_{c} j_{d}\right\rangle$ are the reduced matrix elements of the $T_{1}$ and $T_{2}$ operators, respectively. Owing to the nature of our orbitals, the $T_{1}$ operator is scalar in our calculations but $T_{2}$ will have multi-poles satisfying the triangle conditions $\left|j_{a}-j_{c}\right| \leq k \leq j_{a}+j_{c}$ and $\left|j_{b}-j_{d}\right| \leq k \leq j_{b}+j_{d}$. Following Eq. (3.14), it is evident that the multi-poles satisfying the conditions $l_{a}+l_{c}+k=$ even and $l_{b}+l_{d}+k=$ even will be the dominant contributing multi-poles. Diagrammatic representations of the $T_{1}$ and $T_{2}$ operators are shown in Fig. 1.

The above singles and doubles $\mathrm{CC}$ amplitude equations are solved using the following matrix form

$$
\left\langle\Phi_{0}^{*}|| H_{N}^{e f f} \otimes T^{*} \| \Phi_{0}\right\rangle=0,
$$

where the superscript $\left|\Phi_{0}^{*}\right\rangle$ corresponds to the singles $\left(\left|\Phi_{1}\right\rangle\right)$ and doubles $\left(\left|\Phi_{2}\right\rangle\right)$ excited configurations from $\left|\Phi_{0}\right\rangle$ and $H_{N}^{e f f}=\left(H_{N} e^{T}\right)_{c}^{o p}$ is the effective normal ordered Hamiltonian containing only the connected (c) open (op) terms. Here, $T^{*} \mathrm{~s}$ are the $T_{1}$ and $T_{2}$ operators in the singles and doubles amplitude solving equations respectively.

The excited states $\left(\left|\Psi_{K}(J, \pi)\right\rangle\right)$ having specific total angular momentum $J$ and parity $\pi\left(=(-1)^{l}\right.$ with the $l$ 

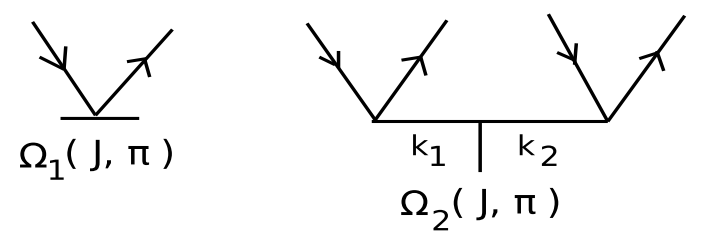

FIG. 2: Diamagnetic representations of the $\Omega_{1}$ and $\Omega_{2}$ EEEOMCC excitation operators. $J$ and $\pi$ are the total angular momentum and parity carried out by the operators. As shown, $J$ value of the $\Omega_{2}$ operator is determined following a triangular condition among two other operators having ranks $k_{1}$ and $k_{2}$.
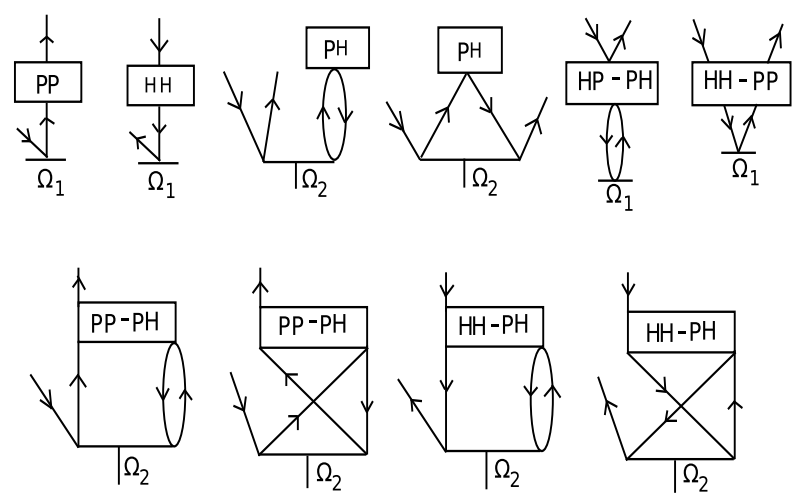

FIG. 3: EE-EOMCC diagrams to determine amplitudes of the $\Omega_{1}$ operators.

orbital quantum number) from the ground state $\left(\left|\Psi_{0}\right\rangle\right)$ of a closed-shell atomic system in an EOMCC method is determined by defining an excitation operator $\Omega_{K}$ as

$$
\left|\Psi_{K}(J, \pi)\right\rangle=\Omega_{K}(J, \pi)\left|\Psi_{0}\right\rangle
$$

where $K=0, K=1, K=2$ etc. correspond to the ground, singly, doubly excited etc. states respectively. Analogous to the CC excitation $T$ operators, we express the $\Omega_{K}$ operators in the second quantized notations as

$$
\begin{aligned}
\Omega_{K} & =\Omega_{1}+\Omega_{2}+\cdots \\
& =\sum_{a, p} \omega_{a}^{p} a^{p^{\dagger}} a_{a}+\frac{1}{4} \sum_{a b, p q} \omega_{a b}^{p q} a^{p^{\dagger}} a^{q^{\dagger}} a_{b} a_{a}+\cdots
\end{aligned}
$$

where $\omega_{a}^{p}, \omega_{a b}^{p q}$, etc. are the amplitudes of the $\Omega_{1}, \Omega_{2}$ etc. operators and obviously, here, $\Omega_{0}=1$. Thus, the eigenvalue equation for the excited states are given by

$$
\begin{array}{r}
H\left|\Psi_{K}\right\rangle=E_{K}\left|\Psi_{K}\right\rangle \\
H \Omega_{K} e^{T}\left|\Phi_{0}\right\rangle=E_{K} \Omega_{K} e^{T}\left|\Phi_{0}\right\rangle .
\end{array}
$$

Following the second quantization notations, we can show that $\Omega_{K}$ and $T$ commute each other. Therefore by operating $e^{-T}$ from the left side of the above equation, we get

$$
\begin{aligned}
e^{-T} H e^{T} \Omega_{K}\left|\Phi_{0}\right\rangle & =E_{K} \Omega_{K}\left|\Phi_{0}\right\rangle \\
\left(e^{-T} H_{N} e^{T}+E_{D F}\right) \Omega_{K}\left|\Phi_{0}\right\rangle & =E_{K} \Omega_{K}\left|\Phi_{0}\right\rangle \\
\left\{\left(H_{N} e^{T}\right)_{c}^{o p}+E_{g}\right\} \Omega_{K}\left|\Phi_{0}\right\rangle & =E_{K} \Omega_{K}\left|\Phi_{0}\right\rangle \\
H_{N}^{e f f} \Omega_{K}\left|\Phi_{0}\right\rangle & =\Delta E_{K} \Omega_{K}\left|\Phi_{0}\right\rangle
\end{aligned}
$$

where $E_{D F}\left(=\left\langle\Phi_{0}|H| \Phi_{0}\right\rangle\right)$ and $E_{g}$ are the DF and ground state energies, respectively. Therefore, $\Delta E_{K}\left(=E_{K}-E_{g}\right)$ corresponds to the excitation energy of the $\left|\Psi_{K}\right\rangle$ state with respect to the ground state. Using the effective Hamiltonian $H_{N}^{e f f}$, we evaluate the excitation energies after projecting $\left\langle\Phi_{L}\right|$ from the left hand side to yield in the following form

$$
\left\langle\Phi_{L}\left|H_{N}^{e f f} \Omega_{K}\right| \Phi_{0}\right\rangle=\Delta E_{K}\left\langle\Phi_{L}\left|\Omega_{K}\right| \Phi_{0}\right\rangle \delta_{L, K},
$$

where $\left|\Phi_{L}\right\rangle$ represents an excited determinantal state with definite values of $J$ and $\pi$. Therefore, $\Omega_{K}$ s have the fixed $J$ and $\pi$ values for which we get

$$
\left\langle\Phi_{L}(J, \pi)\left|H_{N}^{e f f} \Omega_{K}(J, \pi)\right| \Phi_{0}\right\rangle=\Delta E_{L}\left\langle\Phi_{L}(J, \pi)\left|\Omega_{L}(J, \pi)\right| \Phi_{0}\right\rangle .
$$

By applying the completeness principle, the above equation corresponds to

$$
\sum_{K}\left\langle\Phi_{L}(J, \pi)\left|H_{N}^{e f f}\right| \Phi_{K}\right\rangle\left\langle\Phi_{K}\left|\Omega_{K}(J, \pi)\right| \Phi_{0}\right\rangle=\Delta E_{L}\left\langle\Phi_{L}(J, \pi)\left|\Omega_{L}(J, \pi)\right| \Phi_{0}\right\rangle
$$

Considering only the singles $\left(\Omega_{1}\right)$ and doubles $\left(\Omega_{2}\right)$ excitations only, we write down the matrix form as

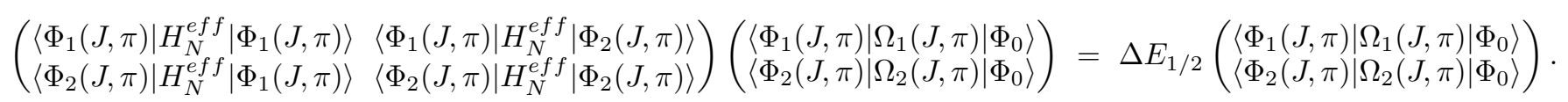




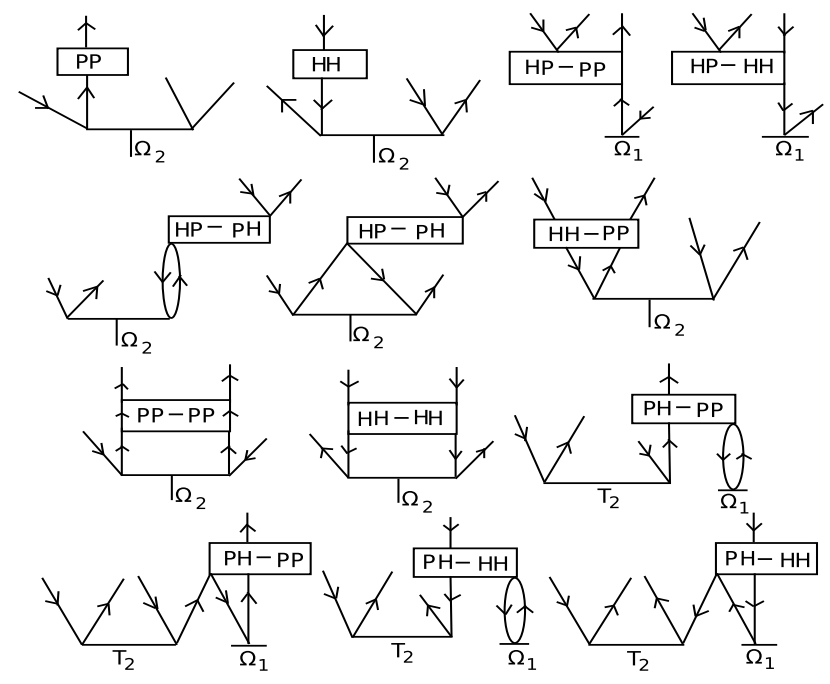

FIG. 4: EE-EOMCC diagrams to determine amplitudes of the $\Omega_{2}$ operators.

the finite matrix elements. We use a modified Davidson algorithm in a iterative scheme to obtain only few roots of the lower eigenvalues as has been applied in [64]. Unlike the $T$ operators, $\Omega_{1}$ has a finite rank equal to $J$ of the state. Similarly, $\Omega_{2}$ has the effective rank equal to $J$ but in contrast to the $T_{2}$ operator, which is obtained from the scalar product of two equal ranked tensor operators, the $\Omega_{2}$ operators are the outcome of general tensor product between two arbitrary ranked tensors. Therefore, its final rank $J$ has been determined using the following types of products

$$
\begin{gathered}
\left\langle J_{1} \pi\left\|\left[t^{k_{1}} u^{k_{2}}\right]^{J}\right\| J_{2} \pi\right\rangle=(2 J+1)^{1 / 2} \sum_{J_{3}}(-1)^{J_{1}+J_{2}+J} \\
\left\{\begin{array}{lll}
k_{1} & k_{2} & J \\
J_{2} & J_{1} & J_{3}
\end{array}\right\}\left\langle J_{1} \pi\left\|t^{k_{1}}\right\| J_{3} \pi\right\rangle\left\langle J_{3} \pi\left\|u^{k_{2}}\right\| J_{2} \pi(4.13)\right.
\end{gathered}
$$

The diagrammatic representation of the $\Omega_{K}$ operators are shown in Fig. 2

To reduce the computational scalability, we divide $H_{N}^{e f f}$ into effective one-body, two-body and three-body terms with two, four and six open lines, respectively. We also make use of the effective two-body terms to construct the effective three-body terms. Using these diagrams the final contributing diagrams to calculate amplitudes for the $\Omega_{1}$ and $\Omega_{2}$ operators are shown in Figs. 3] and 4 , respectively.

\section{RESULTS AND DISCUSSION}

We employ the developed relativistic EE-EOMCC method to calculate energies for many low-lying excited states of the Cr XV, Fe XVII, Co XVIII and Ni XIX ions with different values of the total angular momentum and odd parity. The calculated energies at the CCSD level are reported and compared against the values listed in the national institute of science and technology (NIST) database [65] in Table [1. In order to realize the role of the correlation effects incorporated through the CCSD method, we take the approximation in the effective Hamiltonian as $H_{N}^{e f f} \equiv H_{N}$ and obtain the EEs in the equation-of-motion framework. These results are quoted as second order many-body perturbation theory [MBPT $(2)$ method] results in the same table. We also give EEs estimated from the orbital energies in the same table as the DF results.

As seen from Table I EEs obtained using the DF method are over estimated from the experimental results listed in the NIST database and the $\operatorname{MBPT}(2)$ results are under estimated for all the calculated states in the considered ions. Our CCSD results and the values from NIST are in close agreement and the differences between them are quoted in terms of percentage as $\Delta$ in the above table. From the $\Delta$ values, it is clear that the percentages of accuracies in our calculations are sub-decimal in all the cases.

Among the theoretical calculations, the most recent one is carried out by Aggarwal et al. in which they have employed the multi-configurational Dirac-Fock (MCDF) method using the general-purpose relativistic atomic structure package (GRASP) 66] to calculate the energies along with other properties of Fe XVII ion [67]. Their calculated energies are found to be under estimated in all the considered excited states compared to the NIST data. Bhatia et al. had calculated some of these energies in Fe XVII using a SuperStructure (SS) code in a semi-relativistic approach considering the Breit-Pauli Hamiltonian [68], but their results, when compared with the NIST data, are slightly over estimated and less accurate than our CCSD results. Further, Cornille et al. had also evaluated these energy levels along with few more states using the same SS code [69] but their estimated energies are undervalued than the NIST results. In another work, Sampson et al. had obtained the excitation energies in the Fe XVII ion using a Dirac-Fock-Slater (DFS) atomic code [70] and their results follow a similar trend as obtained by Bhatia et al.. As compared to others, our CCSD results for the EEs in the Fe XVII ion are in close agreement with the values given in the NIST database. However, we do not find calculations of EEs in other ions using the relativistic methods to make a comparative study. Nevertheless, the excellent agreements between our CCSD results and the experimental values against the calculations carried out using other methods demonstrate the potential of the EE-EOMCC method to produce accurate results for the excited states in the considered closed-shell ions.

After achieving high precision calculations of the energies for many transitions in the considered ions, we intend now to estimate the relativistic sensitivity $q$ coefficients for all these transitions. In the Fe XVII ion, we have determined the $q$ parameters for 25 possible inter-combination transitions that are given in Table II. Among them three transitions $2 s^{2} 2 p^{5} 3 s^{3} P_{2}^{o} \rightarrow$ 
$2 s^{2} 2 p^{5} 3 s^{1} P_{1}^{o}, 2 s^{2} 2 p^{5} 3 d{ }^{3} P_{0}^{o} \rightarrow 2 s^{2} 2 p^{5} 3 d^{3} P_{2}^{o}$ and $2 s^{2} 2 p^{5} 3 d{ }^{3} P_{1}^{o} \rightarrow 2 s^{2} 2 p^{5} 3 d{ }^{3} P_{2}^{o}$ lie in the optical regime with the wavelengths $6544.50 \AA, 4460.30 \AA$ and $6849.31 \AA$ respectively. There are also two transitions $2 s^{2} 2 p^{5} 3 s^{3} P_{0}^{o} \rightarrow 2 s^{2} 2 p^{5} 3 s^{3} P_{1}^{o}$ and $2 s^{2} 2 p^{5} 3 d^{3} P_{0}^{o} \rightarrow$ $2 s^{2} 2 p^{5} 3 d^{3} P_{1}^{o}$ that lie near infrared regime while the rest of the transitions fall within ultraviolet (UV) to extreme ultraviolet (EUV) region of the electromagnetic spectrum. It is worth mentioning that the spectra of Fe XVII ion have been extensively studied by many astrophysics groups for investigating different astrophysical plasma, solar plasma, and also in the observation of the absorption lines coming out from various quasars like IRAS $13349+2438$ that are detected by the XMMNewton observatory [43]. Therefore, the above estimated $q$ parameters will serve as the useful ingredients if the astrophysical observations in these lines are directed towards probing temporal variation of the fine structure constant. For the completeness in the understanding of the numerical results for the estimation of the $q$ parameters, we also present the EEs as $\omega(+0.025)$ and $\omega(-0.025)$ in the same table for two different values of $x$ as +0.025 and -0.025 referring to two different values of $\alpha$. The remarkable findings from these results are that we obtain large $q$ parameters with opposite signs in different transitions which is, in fact, a very useful criteria to enhance the effect indicating the variation in $\alpha$ from the observations of these atomic spectra. By analyzing the results of the $q$-parameters in the Fe XVII ion one can find that there are three transitions which could be used as an anchor lines, whose wavelengths are insensitive to the variation of $\alpha$, and transitions having large $q$-parameters can be used as probe lines, whose wavelengths are highly sensitive to variation of $\alpha$ [71]. The $q$ values for these possible anchor lines are $-1660.20,-758$ and 1364.30 in $\mathrm{cm}^{-1}$, whose corresponding laboratory wavelengths lie in the EUV, optical and near infrared (NIR) domain of the electromagnetic spectrum. Observations of these EUV lines from any absorption system will be red-shifted towards the optical range of the spectrum, which could be easily detected using an earth based observatory. Similarly, we estimate $q$ parameters for 15 possible transitions in the Co XVIII ion and present them in Table II. Unlike the case of Fe XVII, the considered transition frequencies in the Co XVIII ion lie only in the UV range. For Co XVIII we find 5 transition with positive $q$-values and the rest 10 transitions correspond to negative $q$-values. Among all the transitions in Co XVIII we can choose $2 s^{2} 2 p^{6}{ }^{1} S_{0} \rightarrow 2 s^{2} 2 p^{5} 3 d^{3} P_{1}^{o}$ transition as an anchor line because of its smaller $q$-value.

In Table III we report the results of the $\alpha$ sensitivity coefficients for the $\mathrm{Cr}$ XV and Ni XIX ions. For both the ions, we have considered 25 possible transitions to estimate the $q$ parameters. In case of $\mathrm{Cr} \mathrm{XV}$, the transition $2 s^{2} 2 p^{5} 3 d^{3} P_{0}^{o} \rightarrow 2 s^{2} 2 p^{5} 3 d^{3} P_{2}^{o}$ lie in the optical region whereas all other transitions fall in the UV range. Out of 25 considered transitions in $\mathrm{Cr} \mathrm{XV}, 8$ of them have positive $q$-values and the rest have neg- ative $q$-values as given in Table III. In this ion, the largest positive and negative $q$-parameters correspond to the transitions $2 s^{2} 2 p^{5} 3 s^{3} P_{1}^{o} \rightarrow 2 s^{2} 2 p^{5} 3 d^{3} P_{0}^{o}$ and $2 s^{2} 2 p^{5} 3 s^{3} P_{2}^{o} \rightarrow 2 s^{2} 2 p^{5} 3 d^{3} P_{0}^{o}$ respectively. At last, we also present the results for the Ni XIX ion in Table III. Similar to the case of Cr XV, Ni XIX ion also has one optical transition $2 s^{2} 2 p^{5} 3 s^{3} P_{2}^{o} \rightarrow 2 s^{2} 2 p^{5} 3 s^{3} P_{1}^{o}$ and rest of the transitions fall in the UV region. It shows that the $q$-values in this ion have the similar trends like in $\mathrm{Cr} \mathrm{XV}$. We have 9 positive and 16 negative $q$-coefficients with the largest positive and negative $q$-values as $104044.80 \mathrm{~cm}^{-1}$ and $-149579.20 \mathrm{~cm}^{-1}$ respectively.

Analogous to the Fe XVII ion, the possible anchor lines in the case of $\mathrm{Cr} \mathrm{XV}$ correspond to wavelengths $19.01 \AA, 7572.31 \AA$ and $11079.10 \AA$ with the $q$-values $-1342.60,-956.40$ and 920.60 , respectively, in $\mathrm{cm}^{-1}$. Moreover in the Ni XIX ion, the transitions $2 s^{2} 2 p^{6}{ }^{1} S_{0} \rightarrow$ $2 s^{2} 2 p^{5} 3 d{ }^{3} P_{2}^{o}, 2 s^{2} 2 p^{5} 3 s{ }^{3} P_{2}^{o} \rightarrow 2 s^{2} 2 p^{5} 3 s{ }^{1} P_{1}^{o}$ and $2 s^{2} 2 p^{5} 3 s^{3} P_{0}^{o} \rightarrow 2 s^{2} 2 p^{5} 3 s^{3} P_{1}^{o}$ which lie in the EUV, optical and NIR region respectively, have small $q$-values and can be chosen as the anchor lines.

\section{CONCLUSION}

We have implemented an equation-of-motion coupledcluster method to calculate the excited states of a closedshell atomic system in the four-component relativistic framework that preserves spherical symmetric properties explicitly. The method has been employed to calculate the excitation energies of four different highly charged ions that are of astrophysical interest. Our calculations are very accurate as compared to their corresponding experimental values. The development will be very useful to study a variety of atomic properties of many atomic systems for which high-precision calculations are in demand. The present method can adequately address the role of the relativistic and the electron correlation effects scrupulously to achieve high precision results to explain many physical problems of modern research interest. To illustrate its potential application, we employ the above method to calculate excitation energies for different values of the fine structure constant in a number of transitions in the considered ions and estimate the relativistic sensitivity coefficients that are of vested interest in the investigation of temporal variation of the fine structure constant using the laboratory astrophysics method. We found large sensitivity coefficients of opposite signs that may be of very crucial information to be analyzed for the detection of enhanced drifts in the course of searching possible variation in the fine structure constant.

\section{Acknowledgment}

We are grateful to B. P. Das and D. Mukherjee for many useful discussions on the theory during the implementation of the code. We thank M. Kallay for providing 
us the diagonalization program used in the code to obtain lower roots of a non-symmetric matrix. The calculations were carried out using the PRL 3TFLOP HPC cluster, Ahmadabad.
[1] J. S. M. Ginges and V. V. Flambaum, Phys. Reports 397, 63 (2004).

[2] M. Pospelov and A. Ritz, Ann. of Phys. 318, 119 (2005).

[3] J. L. Hall, Rev. Mod. Phys. 78, 1279 (2006).

[4] T. W. Hänsch, Rev. Mod. Phys. 78, 1297 (2006).

[5] D. J. Wineland, Rev. Mod. Phys. 85, 1103(2013).

[6] J. P. Uzan, Rev. Mod. Phys. 75, 403 (2003).

[7] H. Chand, R. Srianand, P. Petitjean, B. Aracil, R. Quast, and D. Reimers, Astron. Astrophy. 451, 45 (2006).

[8] M. G. H. Gustavsson and Ann-Mårie Mrtensson-Pendrill, Handbook of Molecular Physics and Quantum Chemistry, vol. 1, part 6, Chapter 30, 477 (ed. S. Wilson, Wiley) (2003).

[9] L. W. Wansbeek, S. Schlesser, B. K. Sahoo, A. E. L. Dieperink, C. J. G. Onderwater, and R. G. E. Timmermans, Phys. Rev. C 86, 015503 (2012).

[10] M. Avgoulea et. al., J. Phys. G: Nucl. Part. Phys. 38, 025104 (2011).

[11] A. Ivanchik et al. Astron. Astrophys. 440, 45 (2005).

[12] J. A. King, M. T. Murphy, W. Ubachs and J. K. Webb, Mon. Not. R. Astron. Soc. 417, 3010 (2011).

[13] B. K. Sahoo, B. P. Das and D. Mukherjee, Phys. Rev. A 79, 052511 (2009).

[14] B. K. Sahoo, Md. R. Islam, B. P. Das, R. K. Chaudhuri and D. Mukherjee, Phys. Rev. A 74, 062504 (2006).

[15] Y. Singh, D. K. Nandy and B. K. Sahoo, Phys. Rev. A 86, 032509 (2012).

[16] B. Arora, D. K. Nandy and B. K. Sahoo, Phys. Rev. A 85, 012506 (2012).

[17] E. Eliav and U. Kaldor, Phys. Rev. A 52, 291 (1995).

[18] A. Borschevsky, V. Pershina, E. Eliav and U. Kaldor, Phys. Rev. A 87, 022502 (2013).

[19] B. K. Mani and D. Angom, Phys. Rev. A 83, 012501 (2011).

[20] I. Shavitt and R. J. Bartlett, Many-Body Methods in Chemistry and Physics, Cambridge University Press, New York (2009).

[21] C.S. Wood et al., Science 275, 1759 (1997).

[22] V. A. Dzuba, V. V. Flambaum and J. S. M. Ginges, Phys. Rev. D 66, 076013 (2002).

[23] W. C. Griffith et al., Phys. Rev. Lett. 102, 101601 (2009).

[24] M. A. Rosenberry and T. E. Chupp, Phys. Rev. Lett. 86, $22(2001)$.

[25] K. V. P. Latha, D. Angom, B. P. Das and D. Mukherjee, Phys. Rev. Lett. 103, 083001 (2009).

[26] Y. Singh, B. K. Sahoo and B. P. Das, Phys. Rev. A 89, 030502(R) (2014).

[27] S. A. Diddams et. al., Science 293, 825 (2001).

[28] P. Gill et. al., Meas. Sci. Technol. 14, 1174 (2003).

[29] P. Gill, Metrologia 42, S125 (2005).

[30] T. Rosenband et. al., Phys. Rev. Lett. 98, 220801 (2007).

[31] T. Rosenband et. al., Science 319, 1808 (2008).

[32] P. Piecuch and R. J. Bartlett, Adv. Quant. Chem. 34, 295 (1999).

[33] J. F. Stanton and R. J. Bartlett, J. Chem. Phys. 98, 7029 (1993).

[34] J. R. Gour, P. Piecuch and M. Wloch, Int. J. Quant.
Chem. 106, 2854 (2006).

[35] D. Mukherjee and P. K. Mukherjee, Chem. Phys. 39, 325 (1979).

[36] H. Pathak, B. K. Sahoo, B. P. Das, N. Vaval and S. Pal, Phys. Rev. A 89, 042510 (2014).

[37] H. Pathak, A. Ghosh, B. K. Sahoo, B. P. Das, N. Vaval and S. Pal, arXiv:1404.7747 (unpublished).

[38] B. Edlén and F. Tyrén, Z. Phys. 101, 206 (1936).

[39] F. Tyrén, Z. Phys. 1011, 314 (1938).

[40] M. Klapisch et al., Phys. Lett. 69A, 34 (1978).

[41] D. L. McKenzie and P. B. Landecker, Astrophys. J. 254, 309 (1982).

[42] M. Audard et al., Astron. Astrophys. 365, L329 (2001).

[43] M. Sako et al., Astron. Astrophys. 365, L168 (2001).

[44] C. Jupén, Mon. Not. Roy. Astron. Soc., Short communication 208, 1 (1984).

[45] K. P. Dere, Astrophys. J. 221, 1062 (1978).

[46] U. Feldman, G. A. Doscheck and J. F. Seely, Mon. Not. Roy. Astron. Soc. 212, 41(1985).

[47] A. K. Dupree, N. S. Brickhouse, G. A. Doscheck, J. C. Green and J. C. Raymond, Astrophys. J. 418, L41 (1993).

[48] J. K. Lepson et al., LLNL-CONF-574833 (2012).

[49] U. Feldman, L. Cohen and M. Swartz, Astrophys. J. 148, 585 (1967).

[50] D. K. Nandy and B. K. Sahoo, Phys. Rev. A 88, 052512 (2013).

[51] D. K. Nandy and B. K. Sahoo, Astron. Astrophys. 563, A25 (2014).

[52] E. Behar, M. Sako, M. Steven and M. Kahn, Astrophys. J. 563, 497 (2001).

[53] P. J. Green et. al., Astrophys. J. 558, 109 (2001).

[54] J.X. Prochaska, J. C. Howk and A. M. Wolfe, Nature 423, 57 (2003).

[55] A. M. Wolfe, D. A. Turnshek, H. E. Smith and R. D. Cohen, Astrophys. J. Suppl. 61249 (1986).

[56] W. L. W. Sargent, C. C. Steidel and A. Boksenberg, Astrophys. J. Suppl. 69, 703 (1989).

[57] P. Molaro et al., Astron. Astrophys. 555, A68 (2013).

[58] S. A. Levshakov, M. Centurión, P. Molaro, and S. D'Odorico, Astron. Astrophys. 434, 827 (2005).

[59] M. T. Murphy, J. K. Webb and V. V. Flambaum, Mon. Not. R. Astron. Soc. 384, 1053 (2008).

[60] V. A. Dzuba, V. V. Flambaum and J. K. Webb, Phys. Rev. A 59, 1 (1998).

[61] V. A. Dzuba, V. V. Flambaum and J. K. Webb, Phys. Rev. Lett. 82, 888 (1999).

[62] M. T. Murphy et al., Lect. Notes Phys. Springer-Verlag, Berlin 648, 131 (2004).

[63] S. A. Levshakov, Lect. Notes Phys., Springer-Verlag, Berlin 648, 151 (2004).

[64] J. B. Yannick et. al., J. Chem. Phys. 122, 154107 (2005).

[65] http://physics.nist.gov/cgi-bin/ASD/energy1.pl

[66] I. P. Grant, B. J. McKenzie, P. H. Norrington, D. F. Mayers and N. C. Pyper, Comput. Phys. Commun. 21, 207 (1980).

[67] K. M. Aggarwal, F. P. Keenan and A. Z. Msezane, As- 
trophys. J. Suppl. 144, 169 (2003)

[68] A. K. Bhatia and J. L. R. Saba, Astrophys. J. 563, 434 (2001).

[69] M. Cornille et al., Astron. Astrophys. Suppl. ser. 105, 77 (1994).
[70] D. H. Sampson, H. L. Zhang, A. K. Mohanty and R. E. Clark, Phys. Rev. A 40, 604 (1989).

[71] M. G. Kozlov, V. A. Korol, J. C. Berengut, V. A. Dzuba and V. V. Flambaum, Phys. Rev. A 70, 062108 (2004). 
TABLE I: Absolutes values of the excitation energies (in $\mathrm{cm}^{-1}$ ) of few low-lying states in the Cr XV, Fe XVII, Co XVIII and Ni XIX ions.

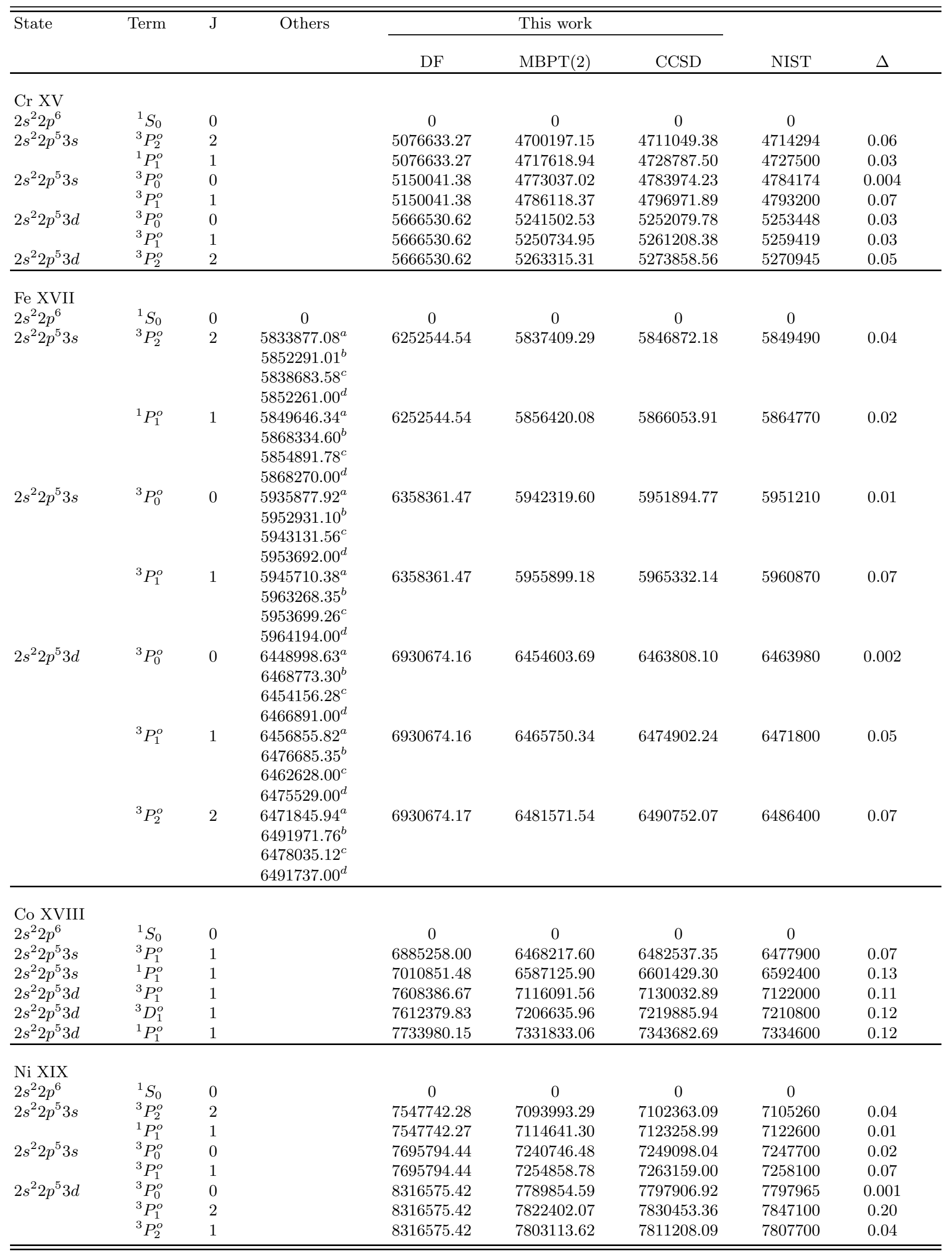


TABLE II: Sensitivity $q$ coefficients (in $\mathrm{cm}^{-1}$ ) for the Fe XVII and Co XVIII ions using the CCSD method. The frequencies $\omega(+0.025)$ and $\omega(+0.025)$ are given as absolute values.

\begin{tabular}{|c|c|c|c|c|c|}
\hline Transitions & $J_{f}$ & $\lambda(\stackrel{\circ}{A})$ & $\omega(+0.025)$ & $\omega(-0.025)$ & $q$ \\
\hline \multicolumn{6}{|l|}{ Fe XVII } \\
\hline $2 s^{2} 2 p^{6}{ }^{1} S_{0} \rightarrow 2 s^{2} 2 p^{5} 3 s^{3} P_{2}^{o}$ & 2 & 17.09 & 5845928.04 & 5847815.39 & -37747.00 \\
\hline$\rightarrow 2 s^{2} 2 p^{5} 3 s^{1} P_{1}^{o}$ & 1 & 17.05 & 5865127.95 & 5866977.40 & -36989.00 \\
\hline$\rightarrow 2 s^{2} 2 p^{5} 3 s^{3} P_{1}^{o}$ & 1 & 16.77 & 5967034.92 & 5963673.14 & 67235.60 \\
\hline$\rightarrow 2 s^{2} 2 p^{5} 3 d^{3} P_{1}^{o}$ & 1 & 15.45 & 6474858.88 & 6474941.91 & -1660.60 \\
\hline$\rightarrow 2 s^{2} 2 p^{5} 3 d^{3} P_{2}^{o}$ & 2 & 15.41 & 6490842.94 & 6490653.18 & -3795.20 \\
\hline $2 s^{2} 2 p^{5} 3 s^{3} P_{2}^{o} \rightarrow 2 s^{2} 2 p^{5} 3 s^{1} P_{1}^{o}$ & 1 & 6544.50 & 19199.91 & 19162.01 & -758.00 \\
\hline$\rightarrow 2 s^{2} 2 p^{5} 3 s^{3} P_{0}^{o}$ & 0 & 983.09 & 107655.55 & 102338.19 & -106347.20 \\
\hline$\rightarrow 2 s^{2} 2 p^{5} 3 s^{3} P_{1}^{o}$ & 1 & 897.83 & 121106.88 & 115857.75 & -104982.60 \\
\hline$\rightarrow 2 s^{2} 2 p^{5} 3 d^{3} P_{0}^{o}$ & 0 & 162.73 & 617721.12 & 616148.30 & -31456.40 \\
\hline$\rightarrow 2 s^{2} 2 p^{5} 3 d^{3} P_{2}^{o}$ & 1 & 160.69 & 628930.84 & 627126.52 & -36086.40 \\
\hline$\rightarrow 2 s^{2} 2 p^{5} 3 d^{3} P_{2}^{o}$ & 2 & 157.00 & 644914.90 & 642837.79 & -41542.20 \\
\hline $2 s^{2} 2 p^{5} 3 s^{1} P_{1}^{o} \rightarrow 2 s^{2} 2 p^{5} 3 s^{3} P_{0}^{o}$ & 0 & 1156.87 & 88455.64 & 83176.18 & -105589.20 \\
\hline$\rightarrow 2 s^{2} 2 p^{5} 3 s^{3} P_{1}^{o}$ & 1 & 1040.58 & 101906.97 & 96695.74 & -104224.60 \\
\hline$\rightarrow 2 s^{2} 2 p^{5} 3 d^{3} P_{0}^{o}$ & 0 & 166.73 & 598521.21 & 596986.29 & -30698.40 \\
\hline$\rightarrow 2 s^{2} 2 p^{5} 3 d^{3} P_{1}^{o}$ & 1 & 164.73 & 609730.93 & 607964.51 & -35328.40 \\
\hline$\rightarrow 2 s^{2} 2 p^{5} 3 d^{3} P_{2}^{o}$ & 2 & 160.16 & 625714.99 & 623675.78 & -40784.20 \\
\hline $2 s^{2} 2 p^{5} 3 s^{3} P_{0}^{o} \rightarrow 2 s^{2} 2 p^{5} 3 s^{3} P_{1}^{o}$ & 1 & 10351.97 & 13451.33 & 13519.56 & 1364.60 \\
\hline$\rightarrow 2 s^{2} 2 p^{5} 3 d^{3} P_{1}^{o}$ & 1 & 192.09 & 521275.29 & 524788.33 & 70260.80 \\
\hline$\rightarrow 2 s^{2} 2 p^{5} 3 d^{3} P_{2}^{o}$ & 2 & 186.85 & 537259.35 & 540499.60 & 64805.00 \\
\hline $2 s^{2} 2 p^{5} 3 s^{3} P_{1}^{o} \rightarrow 2 s^{2} 2 p^{5} 3 d^{3} P_{0}^{o}$ & 0 & 198.76 & 496614.24 & 500290.55 & 73526.2 \\
\hline$\rightarrow 2 s^{2} 2 p^{5} 3 d^{3} P_{1}^{o}$ & 1 & 195.72 & 507823.96 & 511268.77 & 68896.20 \\
\hline$\rightarrow 2 s^{2} 2 p^{5} 3 d^{3} P_{2}^{o}$ & 2 & 190.28 & 523808.02 & 526980.04 & 63440.40 \\
\hline $2 s^{2} 2 p^{5} 3 d^{3} P_{0}^{o} \rightarrow 2 s^{2} 2 p^{5} 3 d^{3} P_{1}^{o}$ & 1 & 12787.72 & 11209.72 & 10978.22 & -4630.00 \\
\hline$\rightarrow 2 s^{2} 2 p^{5} 3 d^{3} P_{2}^{o}$ & 2 & 4460.30 & 27193.78 & 26689.49 & -10085.80 \\
\hline $2 s^{2} 2 p^{5} 3 d^{3} P_{1}^{o} \rightarrow 2 s^{2} 2 p^{5} 3 d^{3} P_{2}^{o}$ & 2 & 6849.31 & 15984.06 & 15711.27 & -5455.80 \\
\hline \multicolumn{6}{|l|}{ Co XVIII } \\
\hline $2 s^{2} 2 p^{6}{ }^{1} S_{0} \rightarrow 2 s^{2} 2 p^{5} 3 s^{3} P_{1}^{o}$ & 1 & 15.43 & 6481449.61 & 6483622.64 & -43460.60 \\
\hline$\rightarrow 2 s^{2} 2 p^{5} 3 s^{1} P_{1}^{o}$ & 1 & 15.16 & 6603472.72 & 6599388.48 & 81684.80 \\
\hline$\rightarrow 2 s^{2} 2 p^{5} 3 d^{3} P_{1}^{o}$ & 1 & 14.04 & 7130003.64 & 7130057.70 & -1081.20 \\
\hline$\rightarrow 2 s^{2} 2 p^{5} 3 d^{3} D_{1}^{o}$ & 1 & 13.86 & 7220232.64 & 7219522.98 & 14193.20 \\
\hline$\rightarrow 2 s^{2} 2 p^{5} 3 d^{1} P_{1}^{o}$ & 1 & 13.63 & 7346134.12 & 7341253.33 & 97615.80 \\
\hline $2 s^{2} 2 p^{5} 3 s^{3} P_{1}^{o} \rightarrow 2 s^{2} 2 p^{5} 3 s^{1} P_{1}^{o}$ & 1 & 873.36 & 122023.11 & 115765.84 & -125145.40 \\
\hline$\rightarrow 2 s^{2} 2 p^{5} 3 d^{3} P_{1}^{o}$ & 1 & 155.25 & 648554.03 & 646435.06 & -42379.40 \\
\hline$\rightarrow 2 s^{2} 2 p^{5} 3 d^{3} D_{1}^{o}$ & 1 & 136.44 & 738783.03 & 735900.34 & -57653.80 \\
\hline$\rightarrow 2 s^{2} 2 p^{5} 3 d^{1} P_{1}^{o}$ & 1 & 116.72 & 864684.51 & 857630.69 & -141076.40 \\
\hline $2 s^{2} 2 p^{5} 3 s^{1} P_{1}^{o} \rightarrow 2 s^{2} 2 p^{5} 3 d^{3} P_{1}^{o}$ & 1 & 188.82 & 526530.92 & 530669.22 & 82766.00 \\
\hline$\rightarrow 2 s^{2} 2 p^{5} 3 d^{3} D_{1}^{o}$ & 1 & 161.70 & 616759.92 & 620134.50 & 67491.60 \\
\hline$\rightarrow 2 s^{2} 2 p^{5} 3 d^{1} P_{1}^{o}$ & 1 & 134.73 & 742661.40 & 741864.85 & -15931.00 \\
\hline $2 s^{2} 2 p^{5} 3 d^{3} P_{1}^{o} \rightarrow 2 s^{2} 2 p^{5} 3 d^{3} D_{1}^{o}$ & 1 & 1126.12 & 90229.00 & 89465.28 & -15274.40 \\
\hline$\rightarrow 2 s^{2} 2 p^{5} 3 d^{1} P_{1}^{o}$ & 1 & 470.36 & 216130.48 & 211195.63 & -98697.00 \\
\hline $2 s^{2} 2 p^{5} 3 d^{3} D_{1}^{o} \rightarrow 2 s^{2} 2 p^{5} 3 d^{1} P_{1}^{o}$ & 1 & 807.75 & 125901.48 & 121730.35 & -83422.60 \\
\hline
\end{tabular}


TABLE III: Sensitivity $q$ coefficients (in $\mathrm{cm}^{-1}$ ) for the Cr XV and Ni XIX ions using the CCSD method. The frequencies $\omega(+0.025)$ and $\omega(+0.025)$ are given as absolute values.

\begin{tabular}{|c|c|c|c|c|c|}
\hline Transitions & $J_{f}$ & $\lambda(\AA)$ & $\omega(+0.025)$ & $\omega(-0.025)$ & $q$ \\
\hline \multicolumn{6}{|l|}{ Cr XV } \\
\hline $2 s^{2} 2 p^{6}{ }^{1} S_{0} \rightarrow 2 s^{2} 2 p^{5} 3 s^{3} P_{2}^{o}$ & 2 & 21.21 & 4710372.93 & 4711725.19 & -27045.20 \\
\hline$\rightarrow 2 s^{2} 2 p^{5} 3 s^{1} P_{1}^{o}$ & 1 & 21.15 & 4727989.51 & 4729293.95 & -26088.80 \\
\hline$\rightarrow 2 s^{2} 2 p^{5} 3 s^{3} P_{1}^{o}$ & 1 & 20.86 & 4798109.43 & 4795838.96 & 45409.40 \\
\hline$\rightarrow 2 s^{2} 2 p^{5} 3 d^{3} P_{1}^{o}$ & 1 & 19.01 & 5261218.88 & 5261286.01 & -1342.60 \\
\hline$\rightarrow 2 s^{2} 2 p^{5} 3 d^{3} P_{2}^{o}$ & 2 & 18.97 & 5273946.73 & 5273762.90 & 3676.60 \\
\hline $2 s^{2} 2 p^{5} 3 s^{3} P_{2}^{o} \rightarrow 2 s^{2} 2 p^{5} 3 s^{1} P_{1}^{o}$ & 1 & 7572.31 & 17616.58 & 17568.76 & -956.40 \\
\hline$\rightarrow 2 s^{2} 2 p^{5} 3 s^{3} P_{0}^{o}$ & 0 & 1431.02 & 74775.43 & 71106.67 & -73375.20 \\
\hline$\rightarrow 2 s^{2} 2 p^{5} 3 s^{3} P_{1}^{o}$ & 1 & 1267.33 & 87736.50 & 84113.77 & -72454.60 \\
\hline$\rightarrow 2 s^{2} 2 p^{5} 3 d^{3} P_{0}^{o}$ & 0 & 185.47 & 541564.39 & 540494.06 & -21406.60 \\
\hline$\rightarrow 2 s^{2} 2 p^{5} 3 d^{3} P_{2}^{o}$ & 1 & 183.44 & 550845.95 & 549560.82 & -25702.60 \\
\hline$\rightarrow 2 s^{2} 2 p^{5} 3 d^{3} P_{2}^{o}$ & 2 & 179.64 & 563573.80 & 562037.71 & -30721.80 \\
\hline $2 s^{2} 2 p^{5} 3 s^{1} P_{1}^{o} \rightarrow 2 s^{2} 2 p^{5} 3 s^{3} P_{0}^{o}$ & 0 & 1764.48 & 57158.85 & 53537.91 & -72418.80 \\
\hline$\rightarrow 2 s^{2} 2 p^{5} 3 s^{3} P_{1}^{o}$ & 1 & 1522.07 & 70119.92 & 66545.01 & -71498.20 \\
\hline$\rightarrow 2 s^{2} 2 p^{5} 3 d^{3} P_{0}^{o}$ & 0 & 190.13 & 523947.81 & 522925.30 & -20450.20 \\
\hline$\rightarrow 2 s^{2} 2 p^{5} 3 d^{3} P_{1}^{o}$ & 1 & 187.99 & 533229.37 & 531992.06 & -24746.20 \\
\hline$\rightarrow 2 s^{2} 2 p^{5} 3 d^{3} P_{2}^{o}$ & 2 & 184.01 & 545957.22 & 544468.95 & -29765.40 \\
\hline $2 s^{2} 2 p^{5} 3 s^{3} P_{0}^{o} \rightarrow 2 s^{2} 2 p^{5} 3 s^{3} P_{1}^{o}$ & 1 & 11079.10 & 12961.07 & 13007.10 & 920.60 \\
\hline$\rightarrow 2 s^{2} 2 p^{5} 3 d^{3} P_{1}^{o}$ & 1 & 210.42 & 476070.52 & 478454.15 & 47672.60 \\
\hline$\rightarrow 2 s^{2} 2 p^{5} 3 d^{3} P_{2}^{o}$ & 2 & 205.43 & 488798.37 & 490931.04 & 42653.40 \\
\hline $2 s^{2} 2 p^{5} 3 s^{3} P_{1}^{o} \rightarrow 2 s^{2} 2 p^{5} 3 d^{3} P_{0}^{o}$ & 0 & 217.27 & 453827.89 & 456380.29 & 51048.00 \\
\hline$\rightarrow 2 s^{2} 2 p^{5} 3 d^{3} P_{1}^{o}$ & 1 & 214.49 & 463109.45 & 465447.05 & 46752.0 \\
\hline$\rightarrow 2 s^{2} 2 p^{5} 3 d^{3} P_{2}^{o}$ & 2 & 209.31 & 475837.30 & 477923.94 & 41732.80 \\
\hline $2 s^{2} 2 p^{5} 3 d^{3} P_{0}^{o} \rightarrow 2 s^{2} 2 p^{5} 3 d^{3} P_{1}^{o}$ & 1 & 16747.61 & 9281.56 & 9066.76 & -4296.00 \\
\hline$\rightarrow 2 s^{2} 2 p^{5} 3 d^{3} P_{2}^{o}$ & 2 & 5715.26 & 22009.41 & 21543.65 & -9315.20 \\
\hline $2 s^{2} 2 p^{5} 3 d^{3} P_{1}^{o} \rightarrow 2 s^{2} 2 p^{5} 3 d^{3} P_{2}^{o}$ & 2 & 8676.03 & 12727.85 & 12476.89 & -5019.20 \\
\hline \multicolumn{6}{|l|}{ Ni XIX } \\
\hline $2 s^{2} 2 p^{6}{ }^{1} S_{0} \rightarrow 2 s^{2} 2 p^{5} 3 s^{3} P_{2}^{o}$ & 2 & 14.07 & 7100997.25 & 7103565.26 & -51360.20 \\
\hline$\rightarrow 2 s^{2} 2 p^{5} 3 s^{1} P_{1}^{o}$ & 1 & 14.03 & 7121845.61 & 7124365.04 & -50388.60 \\
\hline$\rightarrow 2 s^{2} 2 p^{5} 3 s^{3} P_{1}^{o}$ & 1 & 13.78 & 7265582.55 & 7260715.41 & 97342.80 \\
\hline$\rightarrow 2 s^{2} 2 p^{5} 3 d^{3} P_{1}^{o}$ & 2 & 12.74 & 7830543.23 & 7830327.75 & 4309.60 \\
\hline$\rightarrow 2 s^{2} 2 p^{5} 3 d^{3} P_{2}^{o}$ & 1 & 12.80 & 7811084.03 & 7811160.03 & -1520.00 \\
\hline $2 s^{2} 2 p^{5} 3 s^{3} P_{2}^{o} \rightarrow 2 s^{2} 2 p^{5} 3 s^{1} P_{1}^{o}$ & 1 & 5767.01 & 20848.36 & 20799.78 & -971.60 \\
\hline$\rightarrow 2 s^{2} 2 p^{5} 3 s^{3} P_{0}^{o}$ & 0 & 702.05 & 150566.74 & 143087.78 & -149579.20 \\
\hline$\rightarrow 2 s^{2} 2 p^{5} 3 s^{3} P_{1}^{o}$ & 1 & 654.27 & 164585.30 & 157150.15 & -148703.0 \\
\hline$\rightarrow 2 s^{2} 2 p^{5} 3 d^{3} P_{0}^{o}$ & 0 & 144.36 & 696739.51 & 694506.60 & -44658.20 \\
\hline$\rightarrow 2 s^{2} 2 p^{5} 3 d^{3} P_{2}^{o}$ & 2 & 134.80 & 729545.98 & 726762.49 & -55669.8 \\
\hline$\rightarrow 2 s^{2} 2 p^{5} 3 d^{3} P_{2}^{o}$ & 1 & 142.36 & 710086.78 & 707594.77 & -49840.20 \\
\hline $2 s^{2} 2 p^{5} 3 s^{1} P_{1}^{o} \rightarrow 2 s^{2} 2 p^{5} 3 s^{3} P_{0}^{o}$ & 0 & 799.36 & 129718.38 & 122288.00 & -148607.60 \\
\hline$\rightarrow 2 s^{2} 2 p^{5} 3 s^{3} P_{1}^{o}$ & 1 & 738.00 & 143736.94 & 136350.37 & -147731.40 \\
\hline$\rightarrow 2 s^{2} 2 p^{5} 3 d^{3} P_{0}^{o}$ & 0 & 148.07 & 675891.15 & 673706.82 & -43686.60 \\
\hline$\rightarrow 2 s^{2} 2 p^{5} 3 d^{3} P_{1}^{o}$ & 2 & 138.02 & 708697.62 & 705962.71 & -54698.20 \\
\hline$\rightarrow 2 s^{2} 2 p^{5} 3 d^{3} P_{2}^{o}$ & 1 & 145.96 & 689238.42 & 686794.99 & -48868.60 \\
\hline $2 s^{2} 2 p^{5} 3 s^{3} P_{0}^{o} \rightarrow 2 s^{2} 2 p^{5} 3 s^{3} P_{1}^{o}$ & 1 & 9615.38 & 14018.56 & 14062.37 & 876.20 \\
\hline$\rightarrow 2 s^{2} 2 p^{5} 3 d^{3} P_{1}^{o}$ & 2 & 166.83 & 578979.24 & 583674.71 & 93909.40 \\
\hline$\rightarrow 2 s^{2} 2 p^{5} 3 d^{3} P_{2}^{o}$ & 1 & 178.57 & 559520.04 & 564506.99 & 99739.00 \\
\hline $2 s^{2} 2 p^{5} 3 s^{3} P_{1}^{o} \rightarrow 2 s^{2} 2 p^{5} 3 d^{3} P_{0}^{o}$ & 0 & 185.23 & 532154.21 & 537356.45 & 104044.80 \\
\hline$\rightarrow 2 s^{2} 2 p^{5} 3 d^{3} P_{1}^{o}$ & 2 & 169.78 & 564960.68 & 569612.34 & 93033.20 \\
\hline$\rightarrow 2 s^{2} 2 p^{5} 3 d^{3} P_{2}^{o}$ & 1 & 181.95 & 545501.48 & 550444.62 & 98862.80 \\
\hline $2 s^{2} 2 p^{5} 3 d^{3} P_{0}^{o} \rightarrow 2 s^{2} 2 p^{5} 3 d^{3} P_{1}^{o}$ & 2 & 2035.21 & 32806.47 & 32255.89 & -11011.60 \\
\hline$\rightarrow 2 s^{2} 2 p^{5} 3 d^{3} P_{2}^{o}$ & 1 & 10272.21 & 13347.27 & 13088.17 & -5182.00 \\
\hline $2 s^{2} 2 p^{5} 3 d^{3} P_{1}^{o} \rightarrow 2 s^{2} 2 p^{5} 3 d^{3} P_{2}^{o}$ & 1 & 2538.07 & 19459.20 & 19167.72 & 5829.60 \\
\hline
\end{tabular}

